\title{
RESEARCH PROCEEDINGS OF A MEETING OF THE NATAL COASTAL BRANCH OF THE SOUTH AFRICAN SOCIETY OF PHYSIOTHERAPY HELD ON 15 MARCH 1982
}

Interest in research and investigation among the membership of the Natal Coastal Branch increased its momentum in early 1980 following a Branch lecture on research and its implications for the physiotherapist. As a result of this lecture an informal group of members particularly interested in research was formed.

Contact with the group has been maintained on a personal basis and despite the many difficulties associated with research in busy hospital and private practices, a number of projects have been undertaken; these cover a wide range of subjects.

As part of the Branch programme for 1982 the committee decided that one Branch meeting should be devoted to those members who were involved in various forms of research and investigations. This meeting was held in March when details pertaining to ten different projects were presented. These included completed studies, as well as trends arising out of ongoing studies. In addition, the format for several projects about to be commenced were presented.

\section{REFLEXOLOGY}

(Mrs O. Ison)

Mrs Ison is one of a small group of therapists who have been using reflexology (zone therapy) in their practice for several years. Although she has treated a considerable number of cases by this method, only one patient has been referred by a doctor specifically for reflexology. As a consequence Mrs Ison usually uses reflexology in conjunction with conventional physiotherapy. However, she was able to report on some of the results of reflexology obtained with patients who had shown little or no improvement when treated with the usual physiotherapeutic modalities.

Positive responses were found in a group of neurologically impaired patients which included cerebral-vascular accident (C.V.A.), cerebral palsy and Parkinson's syndrome. Improvement was largely attributed to a reduction in muscle tone, though in one case of C.V.A. there was some return of 
speech which had been absent for a number of years. In the case of one child with cerebral palsy there was a pronounced improvement in swallowing as well as a general reduction in muscle tone. Several cases of Bell's palsy showed rapid improvement. Of note were two cases, one complicated by shingles and the other with visual and auditory impairment. In a child with a deficiency of pituitary function, there was a marked increase in appetite and energy following treatment by reflexology, though no increase in growth was noted. Sinusitis appears to respond particularly well, an average of four sessions of reflexology being necessary.

Respiratory tract conditions associated with bronchospasm also showed improvement. This was attributed to the relaxation which occurred after reflexology. Mrs Ison reported on a patient who had been referred for treatment of facial pain. The patient also had "pocketing of the gums", a condition characterised by inflammation, infection and recession of the gums. The condition cleared completely after seven treatments using reflexology

In her conclusions Mrs Ison remarked that reflexology and physiotherapy appear to work well when used together but when reflexology had been used on its own results were often dramatic.

\section{CHEST PHYSIOTHERAPY FOR NEONATAL RESPI- RATORY DISTRESS SYNDROME (Mr P. Gounden)}

This report presented the findings of a comparative study of two methods of chest physiotherapy used in the mana gement of neonatal respirat ory distress syndromes. $\mathrm{Mr}$ Gounden compared the method devised by doctors in 1966 and used since then in King Edward Vlll Hospital, Durban, with a revised method. Blood gas analysis was carried out by using a transcutaneous oxygen monitor. This report is presented in detail elsewhere in this issue.

\section{EFFECTS OF EARLY PHYSIOTHERAPY ON PATIENTS WITH STAB WOUNDS TO THE CHEST \\ (Mrs H. Jenkins)}

This was a report of a student project carried out in $198 \mathrm{I}$. The subjects were African male patients with ages ranging from 16-50 years. All the patients had penetrating injuries to the chest resulting in unilateral haemothorax, pneumothorax or haemopneumothorax.

Patients were divided into two groups (A and B). Both groups received the same physiotherapy regimen but the time of commencement differed. Group A consisted of patients stabbed on Friday evenings who commenced physiotherapy as soon as possible after insertion of the intercostal drain. Group B consisted of patients stabbed on Saturday nights who commenced physiotherapy on the following Monday mornings. Both groups received the same physiotherapy regimen which was given twice a day, each session lasting approximately 30-45 minutes.

Examination of results with five parameters consistently showed that early physiotherapy is beneficial to patients with stabbed chests. Lung function tests indicated the value of physiotherapy in both groups. However, it was found that the patients in group A showed greater improvement than those in group B and had better performance on discharge. In group $A$ the average duration of intercostal drainage was 1,52 days and in group B 2,6 days. There was a difference in the average number of days of hospitalisation for each group. Group A patients were hospitalised for an average of 2,31 days and group $B$ patients for 4,77 days. The incidence of pyrexia above $37,2^{\circ} \mathrm{C}$ was 100 per cent in group $B$ patients and half this in group A. Resolution of the haemothorax, pneumothorax or haemopneumothorax was noted. Twenty per cent of patients in group $A$ had a small residual haemothorax, compared with 60 per cent in group B. Two patients in the latter group required aspiration of the haemothorax before discharge.

\section{SHORT WAVE DIATHERMY IN TIHE TREATMENT OF PELVIC INFLAMMATORY DISEASE}

(Miss R. Muthialu) Th
1981.

This was also a report of a student project carried out in

A study was conducted to ascertain whether patients with acute on chronic pelvic inflammatory disease (P.I.D.) treated with drugs and short-wave diathermy (S.W.D.) show quicker improvement than patients treated with drugs only. Patients are diagnosed as having acute on chronic P.I.D. when their condition is passing from an acute into a chronic state.

After being examined and diagnosed by a gynaecologist, 12 female patients were referred to the Department of Physical Medicine. The paticnts were then randomly allocated to 2 groups (A and B), each group consisting of 6 patients. Pattients who had P.I.D. associated with other conditions such as urinary tract infection or peritonitis, and patients who had surgery for the pelvic inflammatory condition, were not included in the study.

Patients in group A received drug therapy alone, consisting of analgesics and antibiotics. In addition to the drug therapy, patients in group B received S.W.D. This was given by the "cross-fire" method, the intensity being a minimal perceptible heating. Patients were treated once daily at the same time each day. On the first day treatment was administered for 3 minutes each way and the time was increased by 1 minute until a 10 minute each way treatment period was reached. This treatment was then continued until two weeks had elapsed.

Indices used to judge resolution were pain rating (using a numerical rating scale), the degree of abdominal distension and vaginal discharge. All of these were assessed before the respective treatments were commenced.

Patients in both groups were examined by the gynaecologist at the end of the first wcek and again at the end of the second week. Assessments of pain, abdominal distension and vaginal discharge were carried out similarly.

Results obtained after 2 wecks showed that in group A 50 percent of the patients were painfree, there was a mean reduction in abdominal distension of 0.42 centimetres and a clearance of vaginal discharge in 50 per cent. In group B these figures were 66 percent, I, 83 centimetres and 83 percent respectively. Degrees of resolution were assessed as: no response (group $\mathrm{A}=33,6 \%$, group $\mathrm{B}=16.6 \%$ ), partial response (group $\mathrm{A}=50 \%$, Group $\mathrm{B}=16,6 \%$ ) and complete response (group $\mathrm{A}=16,6 \%$. group $\mathrm{B}=66.6 \%$ ).

Due to the small number of patients in this study, data were not subjected to statistical analysis. However, it would appear that the individual presenting clinical features, that is pain, abdominal distension and vaginal discharge, improved more in the patients treated with S.W.D. in addition to drugs. Also, the rate of resolution of the P.I.D. occurred over a shorter period of time in these patients.

\section{MYOFASCIAL PAIN DYSFUNCTION SYNDROME (Mr R. Mitchell)}

Mr Mitchell gave an interim report on his work on myofascial pain dysfunction syndrome (M.P.D.S.) as applied to the bite mechanism. This work has ben carried out in conjunction with a dentist for over three ycars. 
He first described and demonstrated one of the behavioural kinesiology (B.K.) tests he uses in his practice. These tests may be usce to assess the physical, mental and emotional welfare of patients. The demonstration showed how muscles or muscle groups can test "Strong" under some circumstances and the same muscles can test "Weak" under other circumstances, particularly when related to stressful situations.

Mr Mitchell uses B.K. testing in the assessment of his patients with M.P.D.S. Recent research, much of which has orlginated in the United States of America. has indicated that a wide range of symptoms and pain syndromes are associated with a laulty or unbalanced bite mechanism. Some of these are: heaclaches: dizziness: syncope: loss of ballance: neck, shoulder and arm pains; low back pain; thoracic pain and spasm: hip/leg pain and stiffness, and some anxiety states. As many patients with these symptoms are sent for physiotherapy. Mr Mitchell contends that cxamination of the whole of the body structure including the bite mechanism is necessialy to give clues to the source of the trouble, particularly in cases which have not responded to the more usual forms of therapy.

Results obtained from patients in whom adjustments to the bite mechanism had been made. were discussed. If the assessment as described shows a possible M.P.U.S. then $\mathrm{Mr}$ Mitchell makes temporary adjustments to the patient's bite using dental wax placed between the molars on both sides, each stage being assessed by B.K. testing as well as the patient's report of pain relief. When the bite mechanism has been thoroughly assessed and the cause of the dysfunction pinpointed, the patient is supplied with a definitive plastic splint which fits over the molars to balance the bite mechanism. This splint is made by the dentist.

$\mathrm{Mr}$ Mitchell's investigations are ongoing and results available to date show that B.K. testing and careful assessment of the bite mechanism could add a new dimension to the physiotherapist's management of a wide range of troublesomc conditions.

\section{MEASUREMENT OF PAIN}

\section{(Mr C. Liggins)}

$\mathrm{Mr}$ Liggins reported on evaluation of the use of 3 subjective pain-rating scales in 50 African subjects. Some of the findings are reported elsewhere in this issue.

\section{THE ROLES OF PHYSIOTHERAPY IN THE MANAGEMENT OF FRACTURES OF THE SHAFT OF FEMUR}

(Mrs L. Gumede)

Mrs Gumede is to undertake a study of the role of physiotherapy in the management of fractures of the shaft of femur. The proposal for this study was presented in the following summarised form: The rehabilitation of a patient who has sustained a fracture of the shaft of femur is a long drawn-out process and several questions have been posed on this issue:

- Why does rehabilitation take so long?

- How can this lengthy period of rehabilitation be shortened?

- Is enough time allocated to rehabilitation at given treatment sessions?

- How much is being done by the patient on his own?

- Is the patient well enough equipped for self-therapy?

- Is the patient willing to do any work? If not, what are his problems? Can the therapist help to solve these problems? If so, how?

$A_{s}$ it is not possible to provide answers to all the above questions, it is apparent that there is a need to re-evaluate the conventional approach to rehabilitation of the patient with fracture of the shaft of the femur. The hypothesis to be tested is that comprehensive paticnt education, improved motivation and psychological support improve patient compliance and thus facilitate the overall rehabilitation of a patient who has sustained a fracture of the shaft of the femur. 1

The aims of the study are:

1. To assess the importance of factors such as:

1.1 education of the patients regarding their condition, explanation of rehabilitation proccdures and their effects.

1.2 psychological support of patients by the physiotherapist.

1.3 motivation of patients.

2. To assess patient compliance with respect to rehabilitation programmes.

3. To evaluate the effectiveness of the physiotherapeutic procedures.

Patients who have sustained a fracture of the shaft of the femur will be allocated to two groups in random fashion. The first group will be treated along conventional lines, whilst the second group will be counselled in addition to having the conventional treatment. This latter group will be given information on their condition: also an insight into the rationale of the doctor's treatment as well as the physiotherapist's treatment.

A handout has been compiled to help edecate the patient in all aspects of his condition. It will give inlormation on the whole series of events throughout the lime the patient is under treatment. The handout is seen as a means of reinforcing the information given to the pationt verbally.

\section{LASER ACUPUNCTURE \\ (Mr D. Dhanraj and Mr C. Liggins)}

Mr Liggins, who has been working in assuctattion with $\mathrm{Mr}$ Dhanraj, reported on the carly impresisions gaince at al result of the usc of laser acupuncture in a range of painful conditions. This mode of treatment is relatively new in Durban and at present is heing used by two privite practitioners.

Acupuncture is a well-established and integral part of Oricntal medicine. It is used to produce analgesia and anaesthesia, and in the trcatment of a wide lange of organic disorders. It is becoming an increasingly popular form of alternative therapy in Western medicine. This is probably because modern pain research is shedding new light on how acupuncture produces its effects. Currently, theories of acupuncture analgesia are rclated to the Gate Control Theory and the established fact that the body produces endogenous opiate-like substances.

The legality of the use of needle acupuncture by physiotherapists in this country is yet to be clarified. However, as laser acupuncture is a stimulation technique, it falls well into the scope of physiotherapy. Conventional needle acu puncture may be accompanied by discomfort and anxiety on insertion of the needles. Also, the acupuncturist must take precautions to ensure sterility of the needles. Laser acupuncture is totally painless and, being non-invasive, there are no problems regarding sterility.

Laser (Light Amplification by Stimulated Emission of Radiation) is already well established in medicine, but the laser used for acupuncture is quite different to the surgical laser in that its intensity is so low that the tissues cannot be damaged.

The apparatus used is a single quartz fibre laser with a power of $2 \mathrm{mw}$ which is emitted in the red part of the spectrum $(\lambda 0,632, \mu \mathrm{m})$. This allows for maximum tissue penetration $(3-15 \mathrm{~mm})$. The frequency range can be varied between $0,2 \mathrm{~Hz}$ and $5 \mathrm{kHz}$ and pulse durations of between 1 
and 60 seconds can be selected. Irradiation is applied to the tissues by a hand-held stylus with a beam exit of $1 \mathrm{~mm}$. Application is to conventional acupunct ure points, "trigger" points or tender "nodules".

Claims have been made for the use of laser acupuneture in a wide range of clinical conditions, such as dermatology (acne vulgaris, herpes and ulcers), E.N.T. (sinusitis), musculo-skeletal pain syndromes, chronic respiratory conditions (asthma) and rheumatology.

When he initially started using laser stimulation for painful conditions, Mr Dhanraj was particularly impressed with the degree of pain relief obtained and the rate at which this was achieved. Often complete pain relief was obtained in one treatment after a relatively short period of stimulation.

As a result of these early impressions it was decided to compare laser acupuncture with other, more conventional, modalities such as ultrasound, short-wave and microwave diathermy. Fourteen patients with pain arising from a range of musculo-skeletal conditions as diagnosed by doctors were randomly divided into 2 groups, $A$ and $B$. Group A patients were treated by laser stimulation applied to acupuncture points, trigger points or painful "spots". The period of stimulation to each area being 20-25 seconds and the total treatment period was 5-6 minutes. Group B patients were treated with ultrasound, short-wave or microwave, applied singly or in combinations, for example ultrasound and shortwave diathermy, for the usual treatment periods.

A numerical rating scale was used to measure initial pain ¿vels and subsequent pain relief. Results were recorded for the first treatment session only. Patients in group A showed a greater average reduction in pain levels $(68,57 \%)$ than those in group B $(47,57 \%)$. In addition 2 patients in the former group reported complete remission of pain after only a few minutes of treatment and no patient in this group had less than 50 per cent reduction in pain level.

It should be emphasised that this short report is of early impressions gained from the use of laser acupuncture in painful conditions. It is intended to study its use further by conducting a trial comparing its effect with those obtained from other, more conventional, methods of physiotherapy. A greater number of subjects will be used and the trial will be confined to specific pain syndromes.

\section{ELECTROMYOGRAPHY (E.M.G.) BIOFEEDBACK IN THE REHABILITATION OF THE UPPER LIMB IN STROKE PATIENTS}

(Miss A. Rajkoomar)

The following is a summary of the project on E.M.G. biofeedback to be undertaken by Miss Rajkoomar.

Biofeedback is the name given to a set of techniques used in the investigation of learned self-control of physiological activity. This awareness of the internal state of the body is brought about by auditory and/or visual signals displayed to the patient which he can manipulate to reach set goals. This technique inserts a patient's volition into the gap of an open feedback loop - hence the name "biofeedback".

Although the use of these techniques is relatively new, the basic principles have been known for some time. They reflect the fact that feedback of information or knowledge of results is essential for the efficient learning of any skill.

This project entails the use of clinical E.M.G. biofeedback apparatus for the treatment of the upper limb of stroke patients in an experimental group. Muscle activity will be displayed to the patient by means of visual cues (electromyographs) and audit ory signals (increase in volume intensity as a greater number of motor units a re recruited). A control group will receive standardised physiotherapy in accordance with a set protocol. The experimental group will also receive standardised physiotherapy, but E.M.G. biofeedback will be used as an adjunct in this group. The hypothesis to be tested is: that E.M.G. biofeedback is a useful adjunct in the rehabilitation of the upper limb function in stroke patients.

Since the early 1960's E.M.G. biofeedback techniques have been used by physiotherapists, particularly in the management of neuromuscular disorders. Adequatc work has been carried out on the aspect of drop foot in stroke patients and the techniques have been found to be effective in facilitating recovery. However, very little attention has been given to the upper limb. Hence there is a need to study and assess the efficacy of E.M.G. biofeedback in the management of the upper limb in stroke patients.

The history of the management of stroke patients has shown that the upper limb is most resistant to therapy and in many cases hand function is impossible to restore. Should E.M.G. biof eedback prove to be effective it will be a valuable addition to the methods already used in the treatment of the upper limb in stroke patients.

\section{PREDICTION NOMOGRAM OF NORMAL INDIAN MALE AND FEMALE ADULTS}

(Mr A. Khan)

Mr Khan has commenced a study with the full title: Prediction Nomogram of Normal Indian Male and Female Adults, Relating Age and Height to Forced Expiratory Volume (F.E.V.) and Forced Vital Capacity (F.V.C.).

Routine vitalograms on apparently normal Indians show that they have lower expiratory values than those indicated by prediction nomograms. A commonly used nomogram is based on values obtained from North American Caucasians.

During trials involving normal adults, Indians present with lower expiratory values than expected. This is not a true indication of their lung function, as obtained values a re not being compared to a nomogram specific to Indians. Furthermore, in Indian patients the difference between the obtained values and those of the above mentioned nomogram is not a true indication of their condition. Hence there is a need for a prediction nomogram of respiratory function specific to the needs of the South African Indian.

The function of the lung is influenced by age, sex and body size. For people of Caucasian descent there does not appear to be any significant variation in lung function, whichever part of the world they live in. In general, Caucasians tend tor have greater vital capacities than other ethnic groups. Inhabitants of the Indian sub-continent and the people of Polynesian stock have the smallest volumes. Negroid and Mongoloid people appear to occupy an intermediate position. The difference in vital capacity has been attributed to the variations in chest size. For example, studies on Zambian Negroids have shown them to have smaller overall chest dimensions to those of Caucasians.

Briefly, the procedure of this study is as follows:

- Normal Indian male and female subjects in the age range I5-65 years are being selected for participation in the study.

- Each subject is required to complete a questionnaire designed to obtain information of any eardio-respiratory problems they may have. If problems are present, these subjects are eliminated from the study.

- Anthropometric measurements including height, weight and chest size are obtained.

- Each subject is required to perform lung function tests using a Vitalograph.

The data which is being collected will be used to either draw up a nomogram specific to South African Indians, or regression equations will be obtained so that existing nomograms can be adjusted. 